\title{
Atributos químicos e qualidade da matéria orgânica do solo em sistemas de colheita de cana-de-açúcar com e sem queima
}

\author{
Diana Signor(1), Rodrigo Vianei Czycza ${ }^{(2)}$, Débora Marcondes Bastos Pereira Milori(3), \\ Tony Jarbas Ferreira Cunha( ${ }^{(1)}$ e Carlos Eduardo Pellegrino Cerri(2)
}

\begin{abstract}
(1)Embrapa Semiárido, Rodovia BR-428, Km 152, Zona Rural, CEP 56302-970 Petrolina, PE, Brasil. E-mail: diana.signor@embrapa. br, tony.cunha@embrapa.br (2)Universidade de São Paulo, Escola Superior de Agricultura Luiz de Queiroz, Departamento de Ciência do Solo, Avenida Pádua Dias, no 11, CEP 13418-900 Piracicaba, SP, Brasil. E-mail: cepcerri@usp.br, rodrigo_czycza@yahoo.com.br ${ }^{(3)}$ Embrapa Instrumentação, Rua XV de Novembro, no 1.452, Centro, Caixa Postal 74113, CEP 560-970 São Carlos, SP, Brasil. E-mail: debora.milori@embrapa.br
\end{abstract}

Resumo - O objetivo deste trabalho foi avaliar atributos químicos do solo, quantificar os teores e os estoques de $\mathrm{C}$ e $\mathrm{N}$, e avaliar a qualidade química da matéria orgânica do solo em sistemas de colheita de cana-de-açúcar com ou sem queima da palhada. Áreas cultivadas com cana-de-açúcar foram avaliadas com ou sem queima da palhada, aos 6 e aos 12 anos após a última reforma do canavial. Além disso, também foram avaliadas uma área de vegetação nativa e outra cultivada por 19 anos sem queima. $\mathrm{O}$ teor de $\mathrm{C}$ no solo das áreas sem queima foi superior ao daquelas queimadas e inferior ao da área com vegetação nativa. $\mathrm{O}$ estoque de $\mathrm{C}$ a $0,0-0,3 \mathrm{~m}$ de profundidade, na área queimada, foi $22 \%$ menor do que o na área sem queima, aos 6 anos, e $43 \%$ menor, aos 12 anos. Os maiores graus de humificação ocorreram na área sem queima por 19 anos; e os menores, na área com vegetação nativa. Os solos cultivados apresentaram maior concentração de grupos fenólicos e carboxílicos nos ácidos húmicos. A fertilidade do solo aumenta em áreas de cana-de-açúcar sem queima, em razão da matéria orgânica do solo mais humificada e da maior quantidade de grupamentos carboxílicos e fenólicos.

Termos para indexação: cargas dependentes do $\mathrm{pH}$, espectroscopia, estoques de $\mathrm{C}$ e $\mathrm{N}$, fertilidade do solo, fracionamento químico, grau de humificação.

\section{Soil chemical attributes and organic matter quality in sugarcane harvest systems with and without burning}

\begin{abstract}
The objective of this work was to evaluate soil chemical attributes, to quantify soil contents and stocks of $\mathrm{C}$ and $\mathrm{N}$, and to evaluate the chemical quality of soil organic matter in sugarcane harvest systems with or without burning. Cultivated sugarcane areas were evaluated with or without burning of the fields at 6 and 12 years after the last renovation of the sugarcane fields. In addition, a native vegetation area and another one cultivated for 19 years without burning were also evaluated. Soil $\mathrm{C}$ content in the unburnt areas was higher than that of the burnt ones and lower than that of the areas with native vegetation. Carbon stock at the $0.0-0.3-\mathrm{m}$ soil depth in the burnt areas was $22 \%$ lower than in the 6-year unburnt area and $43 \%$ lower in the 12-year area. The highest humification degrees occurred in the 19-year unburnt area; and the lowest, in the native vegetation area. The cultivated soils showed higher concentration of phenolic and carboxylic groups in humic acids. Soil fertility increases in the unburnt sugarcane areas because of their more humified organic matter and of the higher quantity of carboxylic and phenolic groups.
\end{abstract}

Index terms: $\mathrm{pH}$-dependent charges, spectroscopy, $\mathrm{C}$ and $\mathrm{N}$ stocks, soil fertility, chemical fractionation, humification degree.

\section{Introdução}

O Brasil é o maior produtor mundial de canade-açúcar, com aproximadamente nove milhões de hectares cultivados e produção estimada em 659 milhões de toneladas (safra 2014/2015), conforme Acompanhamento... (2014). O sistema de colheita sem queima deposita anualmente, sobre o solo, cerca de 10 a $15 \mathrm{Mg} \mathrm{ha}^{-1}$ de resíduos, o que incrementa os teores de carbono no solo a taxas que variam conforme fatores climáticos, variedade da cana-de-açúcar, idade das plantas, textura do solo, entre outros (Leifeld \& Kögel-Knabner, 2005). Segundo Canellas et al. (2007), os estoques de $\mathrm{C}$ no solo podem aumentar até $40 \%$, na camada superficial, e até $35 \%$ em subsuperfície, em 
razão da adoção do sistema de colheita de cana-deaçúcar sem queima.

Assim, a colheita sem uso do fogo possibilita maior sustentabilidade ao sistema de produção, em virtude da influência da matéria orgânica do solo (MOS) - principal forma de armazenamento de $\mathrm{C}$ no solo - sobre os atributos químicos, físicos e biológicos do solo (Bayer \& Mielniczuk, 2008). Além disso, a presença da palha de cana-de-açúcar sobre a superfície permite aumentar de quatro para oito anos o intervalo entre reformas do canavial (Canellas et al., 2003), o que minimiza em $70 \%$ as perdas de nutrientes essenciais ao desenvolvimento das plantas (Robertson \& Thorburn, 2007) e, portanto, representa também um benefício econômico.

Muitos nutrientes interagem com a MOS, o que influencia a ciclagem e a adsorção dos elementos no solo e evita perdas por lixiviação (Bronick \& Lal, 2005). Essa interação ocorre porque as substâncias húmicas que compõem a MOS apresentam grupos funcionais que geram cargas positivas ou negativas, conforme o pH do solo, e os ácidos fúlvicos são a fração com maior representatividade na geração de cargas (Alvarez-Puebla et al., 2005). Em decorrência disso, a MOS influencia significativamente a capacidade de troca catiônica (CTC), o ponto de carga zero (PCZ) e a complexação de elementos tóxicos no solo, já que representa de 20 a $90 \%$ da CTC total dos solos (Canellas et al., 2003; Alvarez-Puebla et al., 2005; Bayer \& Mielniczuk, 2008).

As substâncias húmicas são os componentes mais estáveis da MOS, constituídas por uma mistura heterogênea de compostos orgânicos altamente polimerizados que, operacionalmente, podem ser subdivididas em ácidos fúlvicos (AF), ácidos húmicos (AH) e humina $(\mathrm{H})$ (Stevenson, 1994), os quais apresentam características químicas, físicas e morfológicas diferenciadas entre si e relacionamse à qualidade da MOS (Canellas et al., 2003). As características químicas da fração $\mathrm{AH}$ podem ser usadas para avaliar indiretamente as alterações da qualidade da MOS em consequência de formas de manejo e tipos de solo (Kalbitz et al., 1999; Dobbss et al., 2009; You et al., 2014). Mudanças no manejo do solo podem influenciar a estabilização e o comportamento dessas substâncias e, consequentemente, afetar o comportamento dos atributos químicos e microbiológicos do solo. Canellas et al. (2003) avaliaram uma área cultivada com cana-de- açúcar, colhida sem queima por 55 anos, e verificaram que esse sistema de colheita promoveu aumento de $157 \%$ nos ácidos húmicos, na camada $0-0,20 \mathrm{~m}$ de profundidade, e de $57 \%$, na camada $0,20-0,40 \mathrm{~m}$, e que esse aumento esteve relacionado ao maior grau de aromaticidade e à diminuição da acidez da MOS.

O objetivo deste trabalho foi avaliar os atributos químicos do solo, quantificar os teores e os estoques de $\mathrm{C}$ e $\mathrm{N}$, e avaliar a qualidade química da matéria orgânica em sistemas de colheita da cana-de-açúcar com e sem queima da palhada.

\section{Material e Métodos}

Áreas comerciais cultivadas com cana-de-açúcar foram avaliadas aos 6 e aos 12 anos, após a última reforma do canavial, em dois sistemas de colheita (com ou sem queima da palhada). Além disso, também foram avaliadas uma área com vegetação nativa, com as mesmas condições de solo, clima e topografia, e outra colhida sem queima por 19 anos - uma das áreas mais antigas sob esse sistema no Brasil. Esta área com 19 anos de cultivo apresenta solo com textura média de apenas $357 \mathrm{~g} \mathrm{~kg}^{-1}$ de argila, na camada $0,0-0,3 \mathrm{~m}$. Assim, ela somente foi incluída nas avaliações de qualidade da MOS, uma vez que ela difere das demais áreas, que apresentavam textura argilosa: vegetação nativa, $637 \mathrm{~g} \mathrm{~kg}^{-1}$; com queima, 6 anos, $687 \mathrm{~g} \mathrm{~kg}^{-1}$; sem queima, 6 anos, $660 \mathrm{~g} \mathrm{~kg}^{-1}$; com queima, 12 anos, 683 $\mathrm{g} \mathrm{kg}^{-1}$; sem queima, 12 anos, $647 \mathrm{~g} \mathrm{~kg}^{-1}$ de argila, na camada $0-0,30 \mathrm{~m}$. Assim, os tratamentos avaliados foram: cana-de-açúcar aos seis anos após a última reforma, colhida sem queima; cana-de-açúcar aos seis anos após a última reforma, colhida com queima; canade-açúcar aos doze anos após a última reforma, colhida sem queima; cana-de-açúcar aos doze anos após a última reforma, colhida com queima; vegetação nativa (VN, Mata Atlântica Ombrófila); cana-de-açúcar aos dezenove anos após a última reforma, colhida sem queima.

O estudo foi realizado em Latossolo Vermelho distrófico, no município de Pradópolis $\left(21^{\circ} 22^{\prime} \mathrm{S}\right.$, $48^{\circ} 03^{\prime} \mathrm{W}$, à altitude de $620 \mathrm{~m}$ ), na região nordeste do estado de São Paulo. O clima, segundo a classificação de Köppen-Geiger, é do tipo Aw: tropical com verão chuvoso e inverno seco, com temperatura média de $22,5^{\circ} \mathrm{C}$ e precipitação média anual de $1.560 \mathrm{~mm}$. O manejo nutricional foi semelhante em todas as áreas 
cultivadas, com aplicação anual de $50 \mathrm{~kg} \mathrm{ha}^{-1}$ de N, $125 \mathrm{~kg} \mathrm{ha}^{-1}$ de $\mathrm{P}_{2} \mathrm{O}_{5}$ e $125 \mathrm{~kg} \mathrm{ha}^{-1}$ de $\mathrm{K}_{2} \mathrm{O}$. Ao plantio, incorporou-se torta de filtro (100 $\mathrm{Mg} \mathrm{ha}^{-1}$ ) (Galdos et al., 2009).

Abriram-se trincheiras em todas as áreas estudadas, em agosto de 2008, para a coleta de amostras de solo. Em cada área, abriu-se uma trincheira central (1,5 $\mathrm{m}$ de comprimento, $0,8 \mathrm{~m}$ de largura, $1,2 \mathrm{~m}$ de profundidade) e quatro minitrincheiras periféricas (0,4 $\mathrm{m}$ de comprimento, $0,4 \mathrm{~m}$ de largura, $0,4 \mathrm{~m}$ de profundidade), distribuídas em um raio de $50 \mathrm{~m}$ em torno da trincheira central. Da trincheira central coletaram-se amostras de solo deformadas e indeformadas, em duas paredes adjacentes, às camadas $0,00-0,10$, $0,10-0,20,0,20-0,30,0,40-0,50,0,50-0,60,0,70-0,80$ e $0,90-1,00 \mathrm{~m}$. Das trincheiras periféricas, coletaramse amostras de solo deformadas e indeformadas em apenas uma das paredes, às camadas $0,00-0,10,0,10$ 0,20 e $0,20-0,30 \mathrm{~m}$. As amostras indeformadas foram utilizadas para a determinação da densidade aparente do solo (Donagema et al., 2011).

As amostras deformadas foram secas ao ar, homogeneizadas, tamisadas em peneira de malha de $2 \mathrm{~mm}$ (terra fina seca ao ar, TFSA) e quarteadas para a sequência de análises químicas e granulométricas, determinação dos teores totais de $\mathrm{C} \mathrm{e} \mathrm{N}$ e fracionamento químico da MOS.

Os teores de $\mathrm{P}$ disponível e de cátions trocáveis foram determinados pelo método da resina trocadora de cátions (Camargo et al., 2009). Os demais atributos químicos do solo, bem como os teores de areia, silte e argila foram determinados conforme Donagema et al. (2011).

Os teores totais de $\mathrm{C}$ e $\mathrm{N}$ nas amostras de solo foram determinados por combustão seca, em analisador elementar FlashEA 1122HT (Thermo Electron Corporation, Delft, The Netherlands). Antes da análise, as amostras de TFSA foram moídas manualmente, em gral de porcelana, e passadas em peneira de malha de $0,150 \mathrm{~mm}$. Para cada camada de solo amostrada, foram calculados os estoques de $\mathrm{C}$ e $\mathrm{N}$, pela multiplicação do teor de $\mathrm{C}$ ou de $\mathrm{N}(\%)$, pela densidade aparente do solo $\left(\mathrm{g} \mathrm{cm}^{-3}\right)$ e pela espessura da camada amostrada (cm). Os estoques de $\mathrm{C}$ e $\mathrm{N}$ foram corrigidos pela massa de solo equivalente, tendo-se considerado como referência a área de VN (Ellert \& Bettany, 1995).

$\mathrm{O}$ fracionamento químico da MOS foi realizado em amostras das camadas $0,00-0,10$ e $0,10-0,20 \mathrm{~m}$, nas áreas de VN e nas áreas cultivadas com cana-de-açúcar com 12 (com e sem queima) e 19 anos (sem queima). Para cada profundidade, formou-se uma amostra composta a partir das cinco amostras simples de cada área.

O fracionamento químico foi realizado segundo a técnica da solubilidade diferencial, em meio ácido ou alcalino, pela separação das frações $\mathrm{AF}, \mathrm{AH}$ e $\mathrm{H}$, conforme Swift (1996). Após extração, as amostras foram liofilizadas, moídas em almofariz de ágar e caracterizadas por fluorescência e por espectroscopia de infravermelho com transformada de Fourier (FT-IR, Fourier transform infrared radiation spectroscopy).

Para as análises de fluorescência, $2 \mu \mathrm{g}$ das amostras de $\mathrm{AH}$ e AF foram dissolvidos em balão de $10 \mathrm{~mL}$, com solução de $\mathrm{NaHCO}_{3}\left(0,05 \mathrm{~mol} \mathrm{~L}^{-1}\right)$, tendo-se ajustado o pH para 8 (Milori et al., 2002), para minimizar o efeito de reabsorção de fluorescência e reduzir interação entre moléculas. Os espectros de emissão de fluorescência, excitação e varredura sincronizada foram medidos em espectrômetro de luminescência LS-50B (Perkin Elmer, New Jersey, USA). A abertura das fendas de entrada e de saída foi de10 $\mathrm{nm}$, e a velocidade de varredura foi de $500 \mathrm{~nm} \mathrm{~min}{ }^{-1}$.

Os espectros de fluorescência foram obtidos nos modos de emissão, conforme Zsolnay et al. (1999) e Milori et al. (2002), e no modo de varredura sincronizada, segundo Kalbitz et al. (1999). Conforme Zsolnay et al. (1999), mediu-se o espectro de emissão com excitação em $240 \mathrm{~nm}$, intervalo de varredura entre 300 e $700 \mathrm{~nm}$ e filtro de $290 \mathrm{~nm}$. A área sob a emissão, entre 580 e $671 \mathrm{~nm}\left(\mathrm{~A}_{4}\right)$, dividida pela área sob a emissão entre 300 e $390 \mathrm{~nm}\left(\mathrm{~A}_{1}\right)\left(\mathrm{A}_{4} / \mathrm{A}_{1}\right)$, foi utilizada como índice de humificação. O grau de humificação dos $\mathrm{AH}$ é diretamente proporcional à área sob o espectro de emissão com excitação de $465 \mathrm{~nm}\left(\mathrm{~A}_{465}\right)$, obtido no intervalo de varredura entre 480 e $700 \mathrm{~nm}$ e com filtro de $290 \mathrm{~nm}$ (Milori et al., 2002). Para a metodologia de Kalbitz et al. (1999), foram obtidos espectros de varredura sincronizada entre 220 e $650 \mathrm{~nm}$, com filtro de $290 \mathrm{~nm}$, tendo-se tomado $55 \mathrm{~nm}\left(\Delta \lambda=\lambda_{\text {em }}-\lambda_{\text {ex. }}\right)$ como diferença constante de comprimento de onda. A avaliação do índice de humificação foi realizada a partir da razão entre as intensidades de fluorescência em 400 e $360 \mathrm{~nm}$, e 470 e $360 \mathrm{~nm}$.

As amostras também foram submetidas a análises por espectroscopia FT-IR, tendo-se formado pastilhas com $1 \mathrm{mg}$ das substâncias húmicas para cada $100 \mathrm{mg}$ 
de brometo de potássio (Stevenson, 1994). Em cada análise, foram realizadas 32 varreduras no intervalo de 4.000 a $400 \mathrm{~nm}$. No momento da leitura dos espectros de FT-IR, procedeu-se à correção automática da linhabase em 4.000, 2.000 e $400 \mathrm{~nm}$, que se igualou a zero. As análises foram realizadas em espectrômetro modelo Spectrum 1000 (Perkin-Elmer, New Jersey, USA). A análise dos resultados consistiu fundamentalmente da correlação, pico a pico, entre o espectro observado e as bandas dos grupos funcionais caracterizados e identificados na literatura (Tabela 1), e a área de VN foi considerada como amostra-padrão.

Os resultados obtidos foram submetidos à análise de variância pelo programa Statistical Analysis Systems (SAS Institute, Cary, NC, EUA), tendo-se considerado como fonte de variação os manejos de colheita e a profundidade de amostragem. As médias foram comparadas pelo teste de Tukey, a 5\% de probabilidade.

\section{Resultados e Discussão}

A acidez ativa do solo aumentou com a profundidade, na área de 12 anos queimada, em razão da baixa adição de resíduos, já que o efeito tampão promovido pelos resíduos garante menor acidificação (Lepsch, 2011) (Tabela 2). Houve redução da CTC total do solo com a profundidade em $\mathrm{VN}$ e na área colhida sem queima há 12 anos, o que reflete o papel da matéria orgânica adicionada por meio da palhada para a formação de grupos funcionais com cargas dependentes de $\mathrm{pH}$ no solo (Brady \& Weil, 2012).

Os maiores teores de $\mathrm{P}$ disponível foram observados nas áreas sem queima, e houve redução em profundidade na $\mathrm{VN}$ e nas áreas queimadas (Tabela 2). Esse comportamento indica que, nas áreas

Tabela 1.Principais bandas do espectro IV da amostra-padrão de ácido húmico $(\mathrm{AH})$, e suas atribuições segundo Canellas (2005).

\begin{tabular}{lc}
\hline Banda & Atribuições \\
\hline $3.500-3.300$ & Estiramento de $\mathrm{OH}$ carboxílico, fenol, álcool ou água \\
$2.850-2.960$ & Estiramento de $\mathrm{C}-\mathrm{H}$ alifáticos \\
$1.700-1.720$ & $\mathrm{C}=\mathrm{O}$ de ácidos carboxílicos, aldeídos e cetonas \\
$1.630-1.700$ & Estiramento de $\mathrm{C}=\mathrm{O}$ de $\mathrm{COO}^{-}$aromáticos, cetonas ou \\
$1.450-1.500$ & $\mathrm{C}=\mathrm{C}$ conjugado com $\mathrm{COO}^{-}$ou amidas \\
$1.200-1.300$ & $\mathrm{C}=\mathrm{C}$ de aromáticos e amidas $\mathrm{NH}_{2}$ ou $\mathrm{NH}$ \\
$730-770$ & Estiramento $\mathrm{C}-\mathrm{O}$ de grupos fenólicos ou ácidos \\
& carboxílicos \\
\hline
\end{tabular}

sem queima, há um importante aporte de $\mathrm{P}$ por meio da palhada, inclusive em profundidade, resultados que concordam com os obtidos por Canellas et al. (2003), que verificaram teores de $\mathrm{P}$ disponível no solo 8,7 vezes maiores nas áreas sem queima do que nas áreas queimadas. Os teores de $\mathrm{K}$ trocável diminuíram em profundidade, exceto na área de 6 anos sem queima.

Os teores de $\mathrm{C}$ na camada superficial das áreas cultivadas foram menores do que os da $\mathrm{VN}$, com reduções de $21 \%$ (na área de 12 anos sem queima) a 48\% (na área de 6 anos queimada) (Tabela 3), o que corrobora as observações de Don et al. (2011). A área de cana-de-açúcar cultivada há 12 anos sem queima apresentou maiores teores de $\mathrm{C}$ do que a área queimada, em todas as profundidades. $\mathrm{Na}$ área de 6 anos, isso ocorreu apenas na camada $0-0,10 \mathrm{~m}$, o que mostra o efeito do manejo da cana-de-açúcar sem queima como uma importante ferramenta para aumentar o conteúdo de $\mathrm{C}$ no solo, em consequência da deposição de palha sobre a superfície. Resultados similares foram obtidos por Signor et al. (2014).

Os teores de $\mathrm{N}$ no solo, na área de 12 anos sem queima, foram similares aos de $\mathrm{VN}(0,0-0,2 \mathrm{~m})$ e foram reduzidos em profundidade na $\mathrm{VN}$ e nas áreas sem queima (Tabela 3), o que reflete a importância da mineralização dos resíduos para a disponibilidade de $\mathrm{N}$ no solo. Luca et al. (2008) avaliaram áreas de cana-deaçúcar com ou sem queima da palhada, em Latossolo Vermelho distroférrico, e observaram teores de $\mathrm{N}$ entre 1,04 e 1,45 $\mathrm{g} \mathrm{kg}^{-1}$, no entanto não verificaram variação dos teores em profundidade, nem diferenças nos teores desse elemento no solo em razão dos sistemas de colheita. No presente trabalho, os teores de $\mathrm{N}$ na área de 12 anos sem queima foram maiores do que na área queimada, o que pode representar menor necessidade de adubação nitrogenada à cana-de-açúcar sem queima da palhada.

A densidade aparente do solo foi utilizada para os cálculos dos estoques de $\mathrm{C}$ e $\mathrm{N}$ (Tabela 3 ). Os maiores estoques de $\mathrm{C}(0,0-0,1$ e $0,0-0,3 \mathrm{~m})$ ocorreram na $\mathrm{VN}$, seguido pela área de 12 anos sem queima, enquanto os menores valores ocorreram nas áreas queimadas. Resultados semelhantes ocorreram para os estoques de $\mathrm{N}(0,0-0,1 \mathrm{~m})$. Don et al. (2011) relatam que a conversão de vegetação nativa para áreas cultivadas reduz de 25 a $30 \%$ os estoques de $\mathrm{C}$ no solo.

$\mathrm{O}$ estoque de $\mathrm{C}(0,0-0,3 \mathrm{~m})$ na área de 12 anos colhida com queima foi $43 \%$ menor do que na área

Pesq. agropec. bras., Brasília, v.51, n.9, p.1438-1448, set. 2016 DOI: 10.1590/S0100-204X2016000900042 
de mesma idade sem queima. Nas áreas de seis anos, o manejo com queima apresentou estoque de $\mathrm{C}$ $(0,0-0,3 \mathrm{~m}) 22 \%$ menor do que na área sem queima, o que corrobora os resultados anteriores. Luca et al. (2008) avaliaram áreas de cana-de-açúcar com quatro anos, em Latossolo Vermelho distroférrico, e verificaram que o estoque de $\mathrm{C}$ na camada $0,0-0,2 \mathrm{~m}$, na área queimada, foi $11 \%$ menor do que na área sem queima. Signor et al. (2014) observaram que, aos seis anos após a última reforma, áreas queimadas apresentam estoques de $\mathrm{C}(0,0-0,2 \mathrm{~m}) 38 \%$ menores do que áreas sem queima. Canellas et al. (2007) mostraram que, após 55 anos de colheita sem queima, o estoque de $\mathrm{C}(0,0-0,2 \mathrm{~m})$ nas áreas queimadas foi $40 \%$ menor do que o da área sem queima.

$\mathrm{O}$ menor grau de humificação dos $\mathrm{AH}$ - caracterizado pela menor estabilidade do $\mathrm{C}$, com predominância de compostos alifáticos, menores concentrações de

Tabela 2. Valores de $\mathrm{pH}$ em água e $\mathrm{CaCl}_{2}$, acidez trocável $(\mathrm{H}+\mathrm{Al})$, teores de fósforo disponível, potássio, cálcio e magnésio trocáveis, capacidade de troca catiônica $\left(\mathrm{CTC}_{\mathrm{T}}\right)$ e saturação por bases $(\mathrm{V} \%)$, em área de vegetação nativa e em áreas cultivadas com cana-de-açúcar, colhidas com ou sem queima da palhada, aos seis e aos doze anos após a última reforma do canavial.

\begin{tabular}{|c|c|c|c|c|c|}
\hline \multirow{2}{*}{$\begin{array}{l}\text { Profundidade } \\
\text { (m) }\end{array}$} & \multirow[t]{2}{*}{ Vegetação nativa } & \multicolumn{2}{|c|}{ Sem queima } & \multicolumn{2}{|c|}{ Com queima } \\
\hline & & 6 anos & 12 anos & 6 anos & 12 anos \\
\hline & \multicolumn{5}{|c|}{$\mathrm{pH}$ água } \\
\hline $0,00-0,10$ & $4,55 \mathrm{Ab}$ & 5,79Aa & $5,74 \mathrm{Aa}$ & $5,67 \mathrm{Aa}$ & $5,72 \mathrm{Ca}$ \\
\hline $0,10-0,20$ & $4,47 \mathrm{Ab}$ & $5,46 \mathrm{Aa}$ & $5,59 \mathrm{Aa}$ & $5,52 \mathrm{Aa}$ & $5,86 \mathrm{Ba}$ \\
\hline \multirow[t]{2}{*}{$0,20-0,30$} & $4,61 \mathrm{Ac}$ & $5,53 \mathrm{Ab}$ & $5,65 \mathrm{Aba}$ & $5,78 \mathrm{Aba}$ & $6,01 \mathrm{Aa}$ \\
\hline & \multicolumn{5}{|c|}{$\mathrm{pH} \mathrm{CalCl}{ }_{2}$} \\
\hline $0,00-0,10$ & $4,06 \mathrm{Ab}$ & $5,16 \mathrm{Aa}$ & $5,04 \mathrm{Aa}$ & $5,03 \mathrm{Aa}$ & $4,91 \mathrm{Ba}$ \\
\hline $0,10-0,20$ & $3,98 \mathrm{Ab}$ & 4,99Aa & $4,94 \mathrm{Aa}$ & $4,84 \mathrm{Aa}$ & $4,97 \mathrm{Ba}$ \\
\hline \multirow[t]{2}{*}{$0,20-0,30$} & $4,05 \mathrm{Ab}$ & $5,00 \mathrm{Aa}$ & $5,02 \mathrm{Aa}$ & $5,15 \mathrm{Aa}$ & $5,18 \mathrm{Aa}$ \\
\hline & \multicolumn{5}{|c|}{$\mathrm{H}+\mathrm{Al}\left(\mathrm{mmol}_{\mathrm{c}} \mathrm{kg}^{-1}\right)$} \\
\hline $0,00-0,10$ & $98,96 \mathrm{Aa}$ & $47,24 \mathrm{Ab}$ & $63,08 \mathrm{Ab}$ & $53,28 \mathrm{Ab}$ & $61,04 \mathrm{Ab}$ \\
\hline $0,10-0,20$ & $85,12 \mathrm{Aa}$ & $51,64 \mathrm{Ab}$ & $65,32 \mathrm{Ab}$ & $56,32 \mathrm{Ab}$ & $55,92 \mathrm{Ab}$ \\
\hline \multirow[t]{2}{*}{$0,20-0,30$} & $70,04 \mathrm{Ba}$ & $49,44 \mathrm{Ab}$ & $58,32 \mathrm{Ab}$ & $43,88 \mathrm{Ab}$ & $44,44 \mathrm{Bb}$ \\
\hline & \multicolumn{5}{|c|}{ P disponível $\left(\mathrm{mg} \mathrm{kg}^{-1}\right)^{(2)}$} \\
\hline $0,00-0,10$ & $14,8 \mathrm{Ab}$ & $28,62 \mathrm{Aa}$ & $20,44 \mathrm{Aab}$ & $23,44 \mathrm{Aab}$ & $14,36 \mathrm{Ab}$ \\
\hline $0,10-0,20$ & $7,90 \mathrm{Bb}$ & $23,40 \mathrm{Aa}$ & $18,18 \mathrm{Aa}$ & $15,26 \mathrm{ABab}$ & $7,85 \mathrm{Bb}$ \\
\hline \multirow[t]{2}{*}{$0,20-0,30$} & $5,94 \mathrm{Bc}$ & $21,28 \mathrm{Aa}$ & $17,54 \mathrm{Aab}$ & $14,68 \mathrm{Bb}$ & $5,72 \mathrm{Bc}$ \\
\hline & \multicolumn{5}{|c|}{$\mathrm{K}^{+}$trocável $\left(\mathrm{mmol}_{\mathrm{c}} \mathrm{kg}^{-1}\right)$} \\
\hline $0,00-0,10$ & $2,02 \mathrm{Ad}$ & $5,70 \mathrm{Ac}$ & $10,70 \mathrm{Ab}$ & $3,20 \mathrm{Ad}$ & $15,32 \mathrm{Aa}$ \\
\hline $0,10-0,20$ & $1,00 \mathrm{Bc}$ & $5,02 \mathrm{Ab}$ & $6,55 \mathrm{Bb}$ & $1,46 \mathrm{Bc}$ & $11,48 \mathrm{Aa}$ \\
\hline \multirow[t]{2}{*}{$0,20-0,30$} & $0,56 \mathrm{Cc}$ & $3,80 \mathrm{Ab}$ & $3,44 \mathrm{Cbc}$ & $0,68 \mathrm{Cc}$ & $6,90 \mathrm{Ba}$ \\
\hline & \multicolumn{5}{|c|}{$\mathrm{Ca}^{2+}$ trocável $\left(\mathrm{mmol}_{\mathrm{c}} \mathrm{kg}^{-1}\right)$} \\
\hline $0,00-0,10$ & $4,54 \mathrm{Ab}$ & $19,66 \mathrm{Aa}$ & $19,25 \mathrm{Aa}$ & $14,85 \mathrm{Aa}$ & $21,04 \mathrm{Aa}$ \\
\hline $0,10-0,20$ & $3,85 \mathrm{Ab}$ & $15,29 \mathrm{Aa}$ & $19,14 \mathrm{Aa}$ & $13,06 \mathrm{Aa}$ & $20,13 \mathrm{Aa}$ \\
\hline \multirow[t]{2}{*}{$0,20-0,30$} & $1,83 \mathrm{Ac}$ & $11,22 \mathrm{Ab}$ & $17,38 \mathrm{Aab}$ & $13,86 \mathrm{Aab}$ & $17,27 \mathrm{Aa}$ \\
\hline & \multicolumn{5}{|c|}{$\mathrm{Mg}^{2+}$ trocável $\left(\mathrm{mmol}_{\mathrm{c}} \mathrm{kg}^{-1}\right)$} \\
\hline $0,00-0,10$ & $4,54 \mathrm{Ac}$ & $11,62 \mathrm{Ab}$ & $11,62 \mathrm{Ab}$ & 7,3Ac & $14,98 \mathrm{Aa}$ \\
\hline $0,10-0,20$ & $1,98 \mathrm{Bc}$ & $8,57 \mathrm{ABab}$ & $10,00 \mathrm{ABab}$ & $5,28 \mathrm{Abc}$ & $14,00 \mathrm{Aa}$ \\
\hline \multirow[t]{2}{*}{$0,20-0,30$} & $0,90 \mathrm{Bd}$ & $6,92 \mathrm{Bbc}$ & $8,74 \mathrm{Bb}$ & $4,92 \mathrm{Bc}$ & $15,52 \mathrm{Aa}$ \\
\hline & \multicolumn{5}{|c|}{$\mathrm{CTC}_{\mathrm{T}}\left(\mathrm{mmol}_{\mathrm{c}} \mathrm{kg}^{-1}\right)$} \\
\hline $0,00-0,10$ & $121,32 \mathrm{Aa}$ & $84,50 \mathrm{Ab}$ & $104,92 \mathrm{Aa}$ & $78,70 \mathrm{Ab}$ & $109,04 \mathrm{Aa}$ \\
\hline $0,10-0,20$ & $102,18 \mathrm{ABa}$ & $80,64 \mathrm{Aa}$ & $101,34 \mathrm{Aa}$ & $76,26 \mathrm{Aa}$ & $101,82 \mathrm{Aa}$ \\
\hline \multirow[t]{2}{*}{$0,20-0,30$} & $80,58 \mathrm{Bab}$ & $71,54 \mathrm{Aab}$ & $88,06 \mathrm{Ba}$ & $63,38 \mathrm{Ab}$ & $88,08 \mathrm{Aa}$ \\
\hline & \multicolumn{5}{|c|}{ Saturação por bases (\%) } \\
\hline $0,00-0,10$ & $18,36 \mathrm{Ac}$ & $44,30 \mathrm{Aa}$ & $40,06 \mathrm{Aab}$ & $32,60 \mathrm{Ab}$ & $43,88 \mathrm{Aa}$ \\
\hline $0,10-0,20$ & $16,60 \mathrm{Ac}$ & $35,68 \mathrm{Aab}$ & $35,72 \mathrm{Aab}$ & $26,66 \mathrm{Abc}$ & $44,42 \mathrm{Aa}$ \\
\hline $0,20-0,30$ & $12,98 \mathrm{Bc}$ & $44,30 \mathrm{Ab}$ & $34,02 \mathrm{Ab}$ & $30,90 \mathrm{Ab}$ & $49,54 \mathrm{Aa}$ \\
\hline
\end{tabular}

${ }^{(1)}$ Médias seguidas de letras iguais, minúsculas nas linhas e maiúsculas nas colunas, não diferem pelo teste de Tukey, a $5 \%$ de probabilidade. ${ }^{(2)} \mathrm{P}$ disponível com o extrator resina. 
Tabela 3. Teores e estoques de carbono e nitrogênio no solo, em área de vegetação nativa e em áreas cultivadas com cana-deaçúcar, com ou sem queima da palhada, aos seis e aos doze anos após a última reforma do canavial ${ }^{(1)}$.

\begin{tabular}{|c|c|c|c|c|}
\hline \multirow[t]{2}{*}{ Área } & \multicolumn{4}{|c|}{ Profundidade de amostragem } \\
\hline & $0,00-0,10 \mathrm{~m}$ & $0,10-0,20 \mathrm{~m}$ & $0,20-0,30 \mathrm{~m}$ & $0,00-0,30 \mathrm{~m}$ \\
\hline & \multicolumn{4}{|c|}{ Teor de $\mathrm{C}\left(\mathrm{g} \mathrm{kg}^{-1}\right)$} \\
\hline Vegetação nativa & $27,7 \pm 4,3 \mathrm{a}$ & $18,5 \pm 0,3 \mathrm{ab}$ & $13,7 \pm 1,6 \mathrm{ab}$ & - \\
\hline Sem queima 6 anos $20,1 \pm 2,1 \mathrm{bc}$ & $15,8 \pm 0,1 \mathrm{abc}$ & $13,3 \pm 0,9 \mathrm{ab}$ & - & \\
\hline Sem queima 12 anos & $21,7 \pm 1,3 b$ & $19,4 \pm 0,1 \mathrm{a}$ & $17,0 \pm 1,2 \mathrm{a}$ & - \\
\hline Com queima 6 anos & $14,4 \pm 1,7 \mathrm{~d}$ & $13,0 \pm 0,3 \mathrm{c}$ & $11,1 \pm 3,8 b$ & - \\
\hline \multirow[t]{2}{*}{ Com queima 12 anos } & $16,3 \pm 1,2 \mathrm{~cd}$ & $14,8 \pm 0,2 \mathrm{bc}$ & $12,1 \pm 1,7 \mathrm{~b}$ & - \\
\hline & \multicolumn{4}{|c|}{ Teor de $\mathrm{N}\left(\mathrm{g} \mathrm{kg}^{-1}\right)$} \\
\hline Vegetação nativa & $2,2 \pm 0,4 \mathrm{a}$ & $1,4 \pm 0,3 \mathrm{ab}$ & $1,0 \pm 0,2 \mathrm{ab}$ & - \\
\hline Sem queima 6 anos & $1,2 \pm 0,2 b$ & $1,0 \pm 0,1 \mathrm{c}$ & $0,9 \pm 0,1 b$ & - \\
\hline Sem queima 12 anos & $1,8 \pm 0,1 \mathrm{a}$ & $1,7 \pm 0,1 \mathrm{a}$ & $1,5 \pm 0,1 \mathrm{a}$ & - \\
\hline Com queima 6 anos & $1,2 \pm 0,1 b$ & $1,1 \pm 0,3 \mathrm{bc}$ & $1,1 \pm 0,5 \mathrm{ab}$ & - \\
\hline \multirow[t]{2}{*}{ Com queima 12 anos } & $1,2 \pm 0,2 b$ & $1,2 \pm 0,2 \mathrm{bc}$ & $1,1 \pm 0,1 \mathrm{ab}$ & - \\
\hline & \multicolumn{4}{|c|}{ Densidade do solo $\left(\mathrm{g} \mathrm{cm}^{-3}\right)$} \\
\hline Vegetação nativa & $0,8 \pm 0,1 \mathrm{c}$ & $1,0 \pm 0,1 \mathrm{c}$ & $1,0 \pm 0,0 \mathrm{~d}$ & - \\
\hline Sem queima 6 anos & $1,2 \pm 0,0 \mathrm{~b}$ & $1,2 \pm 0,1 \mathrm{~b}$ & $1,2 \pm 0,1 \mathrm{c}$ & - \\
\hline Sem queima 12 anos & $1,1 \pm 0,1 b$ & $1,2 \pm 0,1 \mathrm{~b}$ & $1,2 \pm 0,1 \mathrm{c}$ & - \\
\hline Com queima 6 anos & $1,1 \pm 0,1 b$ & $1,1 \pm 0,0 \mathrm{~b}$ & $1,3 \pm 01 b$ & - \\
\hline \multirow[t]{2}{*}{ Com queima 12 anos } & $1,4 \pm 0,1 \mathrm{a}$ & $1,4 \pm 0,1 \mathrm{a}$ & $1,4 \pm 0,1 \mathrm{a}$ & - \\
\hline & \multicolumn{4}{|c|}{ Estoque de $\mathrm{C}\left(\mathrm{Mg} \mathrm{ha}^{-1}\right)$} \\
\hline Vegetação nativa & $23,2 \pm 3,6 \mathrm{a}$ & & & $54,6 \pm 4,9 \mathrm{a}$ \\
\hline Sem queima 6 anos & $12,0 \pm 1,7 \mathrm{bc}$ & & & $36,7 \pm 5,9 \mathrm{bc}$ \\
\hline Sem queima 12 anos & $14,8 \pm 1,4 \mathrm{~b}$ & & & $47,3 \pm 4,0 \mathrm{ab}$ \\
\hline Com queima 6 anos & $9,4 \pm 1,4 \mathrm{~cd}$ & & & $28,6 \pm 4,3 \mathrm{c}$ \\
\hline \multirow[t]{2}{*}{ Com queima 12 anos } & $8,3 \pm 1,0 \mathrm{~d}$ & & & $27,1 \pm 6,8 \mathrm{c}$ \\
\hline & \multicolumn{4}{|c|}{ Estoque de $\mathrm{N}\left(\mathrm{Mg} \mathrm{ha}^{-1}\right)$} \\
\hline Vegetação nativa & $1,9 \pm 0,3 \mathrm{a}$ & & & $4,3 \pm 0,4 \mathrm{a}$ \\
\hline Sem queima 6 anos & $1,1 \pm 0,2 \mathrm{c}$ & & & $3,1 \pm 0,5 \mathrm{ab}$ \\
\hline Sem queima 12 anos & $1,5 \pm 0,1 b$ & & & $4,6 \pm 0,4 \mathrm{a}$ \\
\hline Com queima 6 anos & $1,0 \pm 0,2 \mathrm{c}$ & & & $3,2 \pm 0,5 \mathrm{ab}$ \\
\hline Com queima 12 anos & $1,0 \pm 0,2 \mathrm{c}$ & & & $3,3 \pm 0,8 b$ \\
\hline
\end{tabular}

(1)Para cada atributo, médias seguidas de letras iguais, nas colunas, não diferem pelo teste de Tukey, a $5 \%$ de probabilidade.

Tabela 4. Grau de humificação dos ácidos húmicos e fúlvicos, nas diferentes áreas avaliadas, a diferentes profundidades, por meio de metodologias propostas por: Zsolnay et al. (1999), pela relação $\mathrm{A}_{4} / \mathrm{A}_{1}$; por Kalbitz et al. (1999), pela relação $\mathrm{I}_{465} / \mathrm{I}_{399}$; e por Milori et al. (2002), por meio do comprimento de onda em $\mathrm{A}_{465}$.

\begin{tabular}{|c|c|c|c|c|c|c|}
\hline \multirow[t]{2}{*}{ Área } & \multicolumn{3}{|c|}{ Ácidos húmicos } & \multicolumn{3}{|c|}{ Ácidos fúlvicos } \\
\hline & $\mathrm{A}_{4} / \mathrm{A}_{1}$ & $\mathrm{I}_{465} / \mathrm{I}_{399}$ & $\mathrm{~A}_{465}$ & $\mathrm{~A}_{4} / \mathrm{A}_{1}$ & $\mathrm{I}_{465} / \mathrm{I}_{399}$ & $\mathrm{~A}_{465}$ \\
\hline & \multicolumn{6}{|c|}{$0,00-0,10 \mathrm{~m}$} \\
\hline Vegetação nativa & 0,60 & 2,23 & 119,01 & 0,39 & 0,60 & 54,2 \\
\hline Sem queima 12 anos & 0,83 & 2,47 & 150,95 & 0,42 & 0,68 & 80,40 \\
\hline Com queima 12 anos & 0,84 & 2,52 & 155,04 & 0,41 & 0,69 & 68,54 \\
\hline \multirow[t]{2}{*}{ Sem queima 19 anos } & 1,36 & 3,68 & 212,56 & 0,62 & 1,18 & 111,63 \\
\hline & \multicolumn{6}{|c|}{$0,10-0,20 \mathrm{~m}$} \\
\hline Vegetação nativa & 0,85 & 2,74 & 204,16 & 0,57 & 0,75 & 71,78 \\
\hline Sem queima 12 anos & 0,76 & 2,43 & 159,00 & 0,57 & 0,76 & 80,00 \\
\hline Com queima 12 anos & 0,88 & 2,54 & 156,47 & 0,66 & 0,78 & 81,77 \\
\hline Sem queima 19 anos & 1,17 & 3,03 & 184,85 & 0,86 & 1,04 & 102,77 \\
\hline
\end{tabular}


radicais semiquinona livres e baixa concentração de cadeias aromáticas (Bayer et al., 2002; Milori et al., 2002) - foi observado para a VN (Tabela 4). Este fato ocorreu, provavelmente, pela maior deposição de resíduos vegetais em superfície, de forma contínua, na $\mathrm{VN}$, e promoveu o acúmulo de $\mathrm{C}$ lábil na camada superficial, já que a adição de resíduos é superior à capacidade de processamento pelos microrganismos (Segnini et al., 2013).

Nas áreas cultivadas com cana-de-açúcar, o grau de humificação dos $\mathrm{AH}$ foi semelhante nas camadas 0,0-0,1 e 0,1-0,2 m, independentemente do sistema de colheita (Tabela 3), o que indica que as práticas de manejo do solo, principalmente as relacionadas à reforma do canavial, tornam a MOS homogênea até $20 \mathrm{~cm}$ de profundidade, como relatado por Segnini et al. (2013). Na área sem queima com 12 anos, houve incorporação de $\mathrm{C}$ ao solo, como observado pelo cálculo de estoques de C. Entretanto, a estrutura dos $\mathrm{AH}$ não sofreu grande alterações em razão do manejo de colheita, o que indica que o material lábil é incorporado ao solo, embora ainda não tenha havido tempo suficiente para o manejo afetar a estrutura da matéria orgânica humificada. Nesse sentido, GonzálezPérez et al. (2004) mencionam que há necessidade de um tempo prolongado, para que diferenças no grau de humificação da MOS, em razão do manejo do solo, sejam observadas.

Além disso, nas áreas de 12 anos, o grau de humificação dos $\mathrm{AH}$ foi semelhante entre os sistemas de colheita nas três metodologias (Kalbitz et al., 1999; Zsolnay et al., 1999; Milori et al., 2002). A área sem queima apresentou maior deposição da palhada do que a área queimada, e esperava-se menor grau de humificação. Entretanto, isso não foi observado e pode ser resultado tanto do pouco tempo de adoção desse sistema de colheita quanto do estímulo à atividade microbiana e à degradação da MOS mais recalcitrante, já presente no solo em consequência da deposição do ponteiro da cana-de-açúcar (efeito priming) (Fontaine et al., 2007), que ocorre mesmo em áreas queimadas. Os graus de humificação semelhantes, entre os tratamentos com 12 anos e entre as profundidades, também podem ser explicados pela homogeneização do solo, decorrente das operações de preparo que ocorrem durante a reforma do canavial (Favoretto et al., 2008; Segnini et al., 2013).

A área de 19 anos sem queima apresentou o maior grau de humificação tanto de $\mathrm{AH}$ quanto de $\mathrm{AF}$
(Tabela 4), o que pode estar relacionado à deposição constante de palha sobre a superfície por um longo período de tempo. Nesse sentido, segundo Segnini et al. (2013), nas áreas de cana-de-açúcar, as operações de preparo do solo que ocorrem durante a reforma do canavial favorecem a decomposição dos compostos lábeis dos resíduos, o que resulta em aumento da recalcitrância da MOS.

$\mathrm{O}$ grau de humificação dos $\mathrm{AF}$ foi menor que o dos AH (Tabela 4), em razão de eles serem ácidos compostos de cadeias alifáticas com menores massas moleculares (Stevenson, 1994). Os resultados para os $\mathrm{AH}$ e os $\mathrm{AF}$ foram similares nas metodologias utilizadas, nas duas profundidades avaliadas, o que corrobora os dados de Milori et al. (2002).

Em superfície, os menores graus de humificação dos AF foram verificados para VN (Tabela 4). O grau de humificação de $\mathrm{VN}$ aumentou em profundidade, comportamento típico de sistemas naturais, e foi similar ao grau de humificação dos AF das áreas de cana-deaçúcar de 12 anos. Favoretto et al. (2008) também verificaram aumento da humificação em profundidade, em áreas de vegetação nativa e de plantio direto. $\mathrm{Na}$ área sem queima de 12 anos, independentemente do sistema de colheita, não houve alteração do grau de humificação dos AF em profundidade, o que reflete a homogeneização da MOS, em razão das operações de preparo do solo decorrentes da reforma do canavial. O comportamento observado na área de 19 anos, com maior grau de humificação de AF, pode ser explicado pela ausência de queima e pelo tempo transcorrido desde a última reforma, que favoreceram o acúmulo de $\mathrm{C}$ e a atividade microbiana do solo que levaram à polimerização dos precursores húmicos.

Os espectros de FT-IR dos AH e dos AF, em que a área de VN é considerada como amostra-padrão estão apresentados na Figura 1. Para os AH, nas duas profundidades, houve diferenciação das bandas de $1.260 \mathrm{~nm}$ e $1.680 \mathrm{~nm}$. As amostras provenientes de solos cultivados com cana-de-açúcar apresentaram maior concentração de grupos fenólicos e carboxílicos do que a $\mathrm{VN}$, nas duas profundidades, resultados que concordam com os obtidos por Bayer et al. (2002), Milori et al. (2002) e González-Pérez et al. (2004).

A área de 19 anos sem queima apresentou maior concentração de ácidos carboxílicos e grupamentos fenólicos nos $\mathrm{AH}$ do que as demais áreas cultivadas, o que pode ser explicado pelo maior grau de humificação 


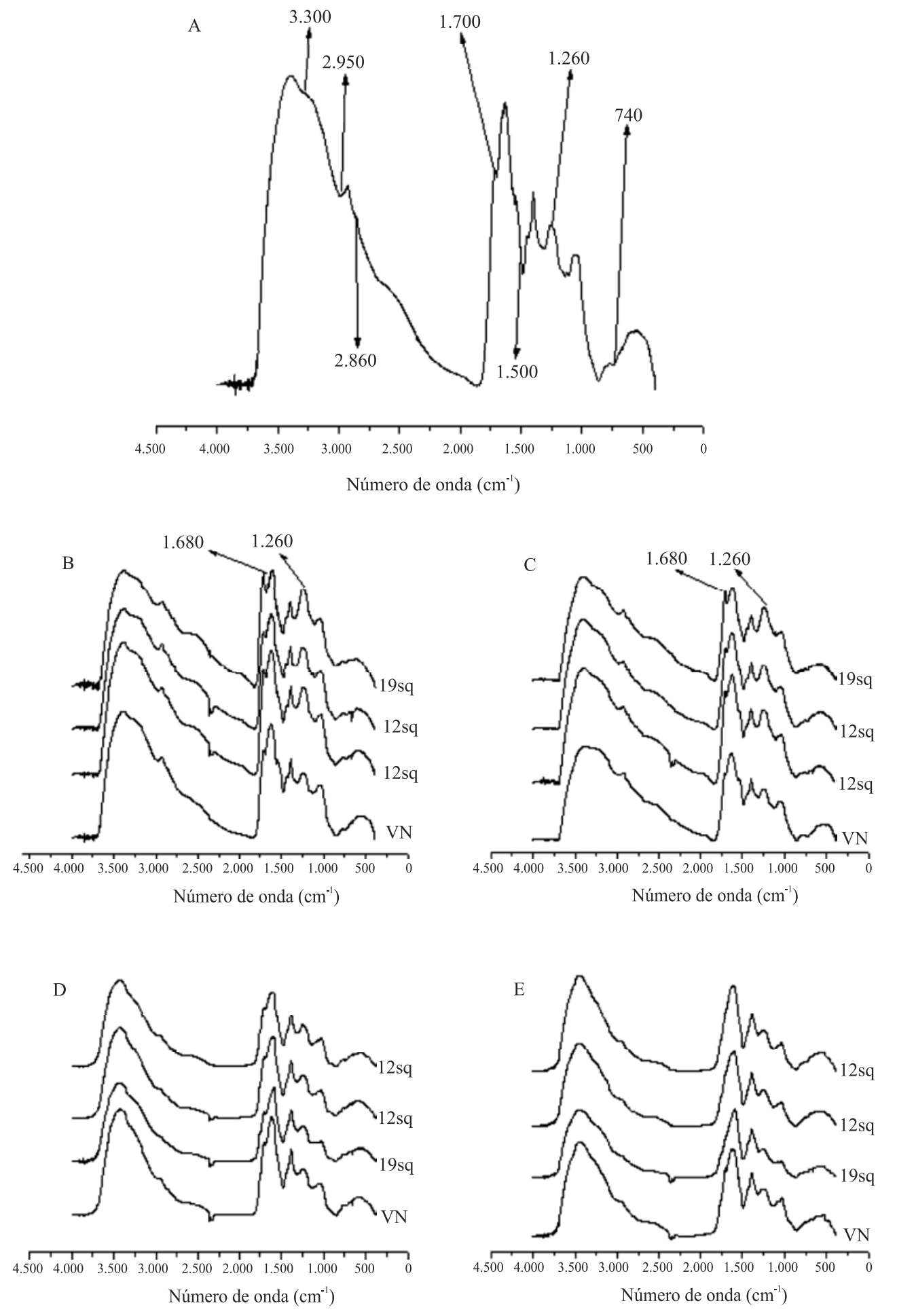

Figura 1. Espectroscopia de infravermelho, em amostras de solo na área de vegetação nativa, com as respectivas bandas de absorção (A). Espectroscopia de Infravermelho para amostras de ácidos húmicos (AH), nas camadas de 0,0-0,1 (B) e 0,1-0,2 m (C); e para amostras de ácidos fúlvicos (AF), nas camadas de 0,0-0,1 (D) e 0,1-0,2 m (E); nas áreas de 12 anos sem queima (12sq) e com queima (12cq), 19 anos sem queima (19sq) e vegetação nativa (VN). 
(Tabela 4). Canellas et al. (2007) avaliaram a qualidade da MOS, em áreas de cana-de-açúcar manejadas sem queima da palhada por 55 anos, e verificaram grau de aromaticidade quatro vezes maior do que nas áreas queimadas, bem como aumento da concentração de $\mathrm{AH}$ mais humificados na área de cana-crua. $\mathrm{O}$ aumento da aromaticidade é uma das principais transformações químicas decorrentes do aumento da humificação e da estabilização da MOS (Stevenson, 1994; Milori et al., 2002). Além disso, nos ácidos carboxílicos e grupamentos fenólicos, ocorre a geração de cargas negativas e, portanto, esses grupamentos influenciam a retenção de nutrientes no solo, considerada como benefício de alta aplicabilidade nos solos com altos teores de caulinita, como os brasileiros (Stevenson, 1994).

Nas áreas de 12 anos, não se verificaram diferenças entre as concentrações de ácidos carboxílicos e grupamentos fenólicos nos AH em razão do sistema de colheita (Figuras $1 \mathrm{~B}$ e $1 \mathrm{C}$ ), o que corrobora o grau de humificação observado nas duas profundidades (Tabela 4). Esses resultados concordam com os valores de CTC total observados nas áreas de 12 anos com e sem queima (Tabela 1), às camadas $0,0-0,1 \mathrm{e}$ $0,1-0,2 \mathrm{~m}$, para as quais não houve diferença entre os sistemas de colheita.

Para os AF, em ambas as profundidades, não se observaram diferenças nos espectros (Figura $1 \mathrm{D}$ e $1 \mathrm{E}$ ). As concentrações de ácidos carboxílicos e grupamentos fenólicos nos AF foram menores do que nos $\mathrm{AH}$, nas duas profundidades, o que pode ser decorrente do menor grau de humificação dos AF, assim como da proteção do $\mathrm{C}$ pelos microagregados do solo e de fortes ligações com os argilominerais, que tornaram os $\mathrm{AH}$ mais estáveis e protegidos. Dick et al. (2003) observaram a mesma tendência ao avaliar o comportamento dos espectros em Latossolo e em Chernossolo. Dobbss et al. (2009), avaliaram os AH e AF de sete Latossolos e observaram que os AH são mais hidrofóbicos e com maior conteúdo de compostos aromáticos do que os $\mathrm{AF}$, o que se deve ao elevado conteúdo de grupamentos oxigenados (-COOH, $-\mathrm{OCH}_{3}$ e $-\mathrm{C}=\mathrm{O}$ ) nos AF. A predominância dos grupamentos alifáticos para os $\mathrm{AF}$ pode ser decorrente da presença e da reposição periódica de materiais no solo que contêm polissacarídeos de origem vegetal, como ligninas e celulose.

\section{Conclusões}

1. A colheita sem queima da palhada melhora a qualidade química e aumenta o teor de $\mathrm{N}$ no solo, o que representa benefícios nutricionais para a cultura da cana-de-açúcar.

2. Nas áreas cultivadas com cana-de-açúcar sem queima da palhada, os estoques de $\mathrm{C}$ e $\mathrm{N}$ no solo são maiores do que nas áreas queimadas; este fato colabora para a sustentabilidade do sistema de produção e, ainda, proporciona benefícios ambientais, como o da redução da pegada de carbono da cultura.

3. Áreas de cana-de-açúcar manejadas por longos períodos (maior que 19 anos) sob o sistema de colheita sem queima apresentam a matéria orgânica do solo com maior grau de humificação e maior quantidade de grupamentos carboxílicos e fenólicos, o que contribui para o aumento da fertilidade desses solos.

4. Após 12 anos de reforma do canavial, o grau de humificação da matéria orgânica do solo não difere entre os sistemas de colheita com ou sem queima dos resíduos.

\section{Agradecimentos}

À Fundação de Apoio à Pesquisa do Estado de São Paulo (Fapesp) e ao Conselho Nacional de Desenvolvimento Científico e Tecnológico (CNPq), pelo apoio financeiro.

\section{Referências}

ACOMPANHAMENTO DA SAFRA BRASILEIRA [DE] CANADE-AÇÚCAR: safra 2014/2015: segundo levantamento, Brasília, DF, v.1, n.2, ago. 2014. Disponível em: <http://www.conab.gov. br/OlalaCMS/uploads/arquivos/14_08_07_08_58_49_boletim_ cana_portugues_-_2o_lev_-_2014-15.pdf $>$. Acesso em: 27 ago. 2014.

ALVAREZ-PUEBLA, R.A.; GOULET, P.J.G.; GARRIDO, J.J. Characterization of the porous structure of different humic fractions. Colloids and Surfaces A: Physicochemical and Engineering Aspects, v.256, p.129-135, 2005. DOI: 10.1016/j. colsurfa.2004.12.062.

BAYER, C.; MIELNICZUK, J. Dinâmica e função da matéria orgânica. In: SANTOS, G. de A.; SILVA, L.S. da; CANELLAS, L.P.; CAMARGO, F. de O. (Ed.). Fundamentos da matéria orgânica do solo: ecossistemas tropicais e subtropicais. 2.ed. rev. e atual. Porto Alegre: Metrópole, 2008. p.7-18.

BAYER, C.; MIELNICZUK, J.; MARTIN-NETO, L.; ERNANI, P.R. Stocks and humification degree of organic matter fractions as 
affected by no-tillage on a subtropical soil. Plant and Soil, v.238, p.133-140, 2002. DOI: 10.1023/A:1014284329618.

BRADY, N.C.; WEIL, R.R. Elementos da natureza e propriedades dos solos. 3.ed. Porto Alegre: Bookman, 2012.716p.

BRONICK, C.J.; LAL, R. Soil structure and management: a review. Geoderma, v.124, p.3-22, 2005. DOI: 10.1016/j. geoderma.2004.03.005.

CAMARGO, O.A. de; MONIZ, A.C.; JORGE, J.A.; VALADARES, J.M.A.S. Métodos de análise química, mineralógica e física de solos do Instituto Agronômico de Campinas. Campinas: Instituto Agronômico, 2009. 77p.

CANELLAS, L.P. Humosfera: tratado preliminar sobre a química das substâncias húmicas. Rio de Janeiro: Ed. do Autor, 2005. 310p.

CANELLAS, L.P.; BALDOTTO, M.A.; BUSATO, J.G.; MARCIANO, C.R.; MENEZES, S.C.; SILVA, N.M. da; RUMJANEK, V.M.; VELlOSO, A.C.X.; SIMÕES, M.L.; MARTIN-NETO, L. Estoque e qualidade da matéria orgânica de um solo cultivado com cana-de-açúcar por longo tempo. Revista Brasileira de Ciência do Solo, v.31, p.331-340, 2007. DOI: 10.1590/S0100-06832007000200015.

CANELLAS, L.P.; VELLOSO, A.C.X.; MARCIANO, C.R.; RAMALHO, J.F.G.P.; RUMJANEK, V.M.; REZENDE, C.E.; SANTOS, G.A. Propriedades químicas de um Cambissolo cultivado com cana-de-açúcar, com preservação do palhiço e adição de vinhaça por longo tempo. Revista Brasileira de Ciência do Solo, v.27, p.935-944, 2003. DOI: 10.1590/S010006832003000500018.

DICK, D.P.; SANTOS, J.H.Z.; FERRANTI, E.M. Chemical characterization and infrared spectroscopy of soil organic matter from two Southern Brazilian soils. Revista Brasileira de Ciência do Solo, v.27, p.29-39, 2003. DOI: 10.1590/S010006832003000100004.

DOBBSS, L.B.; RUMJANECK, V.M.; BALDOTTO, M.A.; VELLOSO, A.C.X.; CANELLAS, L.P. Caracterização química e espectroscópica de ácidos húmicos e fúlvicos isolados da camada superficial de Latossolos brasileiros. Revista Brasileira Ciência do Solo, v.33, p.51-63, 2009. DOI: 10.1590/S010006832009000100006.

DON, A.; SCHUMACHER, J.; FREIBAUER, A. Impact of tropical land-use change on soil organic carbon stocks - a metaanalysis. Global Change Biology, v.17, p.1658-1670, 2011. DOI: 10.1111/j.1365-2486.2010.02336.x.

DONAGEMA, G.K.; CAMPOS, D.V.B. de; CALDERANO, S.B.; TEIXEIRA, W.G.; VIANA, J.H.M. (Org.). Manual de métodos de análise de solo. 2.ed. rev. Rio de Janeiro: Embrapa Solos, 2011. 230p. (Embrapa Solos. Documentos, 132).

ELLERT, B.H.; BETTANY, J.R. Calculation of organic matter and nutrients stored in soils under contrasting management regimes. Canadian Journal of Soil Science, v.75, p.529-538, 1995. DOI: 10.4141/cjss95-075.

FAVORETTO, C.M.; GONÇALVES, D.; MILORI, D.M.B.P.; ROSA, J.A.; LEITE, W.C.; BRINATTI, A.M.; SAAB, S. da C. Determinação da humificação da matéria orgânica de um Latossolo e de suas frações organo-minerais. Química
Nova, v.31, p.1994-1996, 2008. DOI: 10.1590/S010040422008000800015.

FONTAINE, S.; BAROT, S.; BARRÉ, P.; BDIOUI, N.; MARY, B.; RUMPEL, C. Stability of organic carbon in deep soil layers controlled by fresh carbon supply. Nature, v.450, p.277-281, 2007. DOI: $10.1038 /$ nature 06275 .

GALDOS, M.V.; CERRI, C.C.; CERRI, C.E.P. Soil carbon stocks under and unburned sugarcane in Brazil. Geoderma, v.153, p.347352, 2009. DOI: 10.1016/j.geoderma.2009.08.025.

GONZÁLEZ-PÉREZ, M.; MARTIN-NETO, L.; SAAB, S.C.; NOVOTNY, E.H.; MILORI, D.M.B.P.; BAGNATO, V.S.; COLNAGO, L.A.; MELO, W.J.; KNICKER, H. Characterization of humic acids from a Brazilian Oxisol under different tillage systems by EPR, 13C NMR, FTIR and fluorescence spectroscopy. Geoderma, v.118, p.181-190, 2004. DOI: 10.1016/S00167061(03)00192-7.

KALBITZ, K.; GEYER, W.; GEYER, S. Spectroscopic properties of dissolved humic substances - a reflection of land use history in a fen area. Biogeochemistry, v.47, p.219-238, 1999. DOI: 10.1023/A:1006134214244.

LEIFELD, J.; KÖGEL-KNABNER, I. Soil organic matter fractions as early indicators for carbon stock changes under different land-use? Geoderma, v.124, p.143-155, 2005. DOI: 10.1016/j. geoderma.2004.04.009.

LEPSCH, I.F. 19 lições de pedologia. São Paulo: Oficina de Textos, 2011. 456p.

LUCA, E.F. de; FELLER, C.; CERRI, C.C.; BARTHÈS, B.; CHAPLOT, V.; CAMPOS, D.C.; MANECHINI, C. Avaliação de atributos físicos e estoques de carbono e nitrogênio em solos com queima e sem queima de canavial. Revista Brasileira Ciência do Solo, v.32, p.789-800, 2008. DOI: 10.1590/S010006832008000200033.

MILORI, D.M.B.P.; MARTIN-NETOL, L.; BAYER, C.; MIELNICZUK, J.; BAGNATO, V.S. Humification degree of soil humic acid determined by fluorescence spectroscopy. Soil Science, v.167, p.739-749, 2002. DOI: 10.1097/00010694-20021100000004.

ROBERTSON, F.A.; THORBURN, P.J. Management of sugarcane harvest residues: consequences for soil carbon and nitrogen. Australian Journal of Soil Research, v.45, p.13-23, 2007. DOI: 10.1071/SR06080.

SEGNINI, A.; CARVALHO, J.L.N.; BOLONHEZI, D.; MILORI, D.M.B.P.; SILVA, W.T.L. da; SIMÕES, M.L.; CANTARELLA, H.; MARIA, I.C. de; MARTIN-NETO, L. Carbon stock and humification index of organic matter affected by sugarcane straw and soil management. Scientia Agricola, v.70, p.321-326, 2013. DOI: $10.1590 / \mathrm{S} 0103-90162013000500006$.

SIGNOR, D.; ZANI, C.F.; PALADINI, A.A.; DEON, M.D.; CERRI, C.E.P. Estoques de carbono e qualidade da matéria orgânica do solo em áreas cultivadas com cana-de-açúcar. Revista Brasileira de Ciência do Solo, v.38, p.1402-1410, 2014. DOI: 10.1590/S0100-06832014000500005.

STEVENSON, F.J. Humus chemistry: genesis, composition, reactions. $2^{\text {nd }}$ ed. New York: Wiley, 1994. 491p.

Pesq. agropec. bras., Brasília, v.51, n.9, p.1438-1448, set. 2016 DOI: 10.1590/S0100-204X2016000900042 
SWIFT, R.S. Organic matter characterization. In: SPARK, K.M.; PAGE, A.L.; HELMKE, P.A.; LOEPPERT, R.H.; SOLTANPOUR, P.N.; TABATABAI, M.A.; JOHNSTON, C.T.; SUMMER, M.E. (Ed.). Methods of soil analysis. Part 3. Chemical methods. Madison: Soil Science Society of America, 1996. p.1011-1069. (SSSA book series, 5).

YOU, M.; BURGER, M.; LI, L.; ZOU, W.; LI, N.; QIAO, Y.; HAN, $\mathrm{X}$. Changes in soil organic carbon and carbon fractions under different land use and management practices after development from parent material of mollisols. Soil Science, v.179, p.205-210, 2014. DOI: 10.1097/SS.0000000000000059.

ZSOLNAY, A.; BAIGAR, E.; JIMENEZ, M.; STEINWEG, B.; SACCOMANDI, F. Differentiating with fluorescence spectroscopy the sources of dissolved organic matter in soils subjected to drying. Chemosphere, v.38, p.45-50, 1999. DOI: 10.1016/S00456535(98)00166-0.

Recebido em 27 de agosto de 2015 e aprovado em 16 de fevereiro de 2016 